\title{
Prevalência de transtornos fonológicos em crianças do primeiro ano do ensino fundamental
}

\author{
Prevalence of phonological disorders in first grade students of \\ elementary school
}

\author{
Luiza Augusta Rosa Rossi-Barbosa ${ }^{1}$, Antônio Prates Caldeira ${ }^{2}$, Rodrigo Honorato-Marques ${ }^{3}$, Reila Freitas Silva ${ }^{3}$
}

\begin{abstract}
RESUMO
Objetivo: Identificar a prevalência de transtornos fonológicos e verificar a sensibilidade e especificidade do Teste de Rastreamento de Distúrbios Articulatórios de Fala (TERDAF), a partir de sua adaptação, em escolares do primeiro ano do ensino fundamental de Montes Claros, MG (Brasil). Métodos: As crianças foram alocadas de forma aleatória em amostragem por conglomerados em 56 escolas públicas no município. Foram avaliadas 587 crianças com média de idade de 6 anos e 6 meses por meio do TERDAF adaptado e, para verificar a sensibilidade e especificidade do teste, 229 delas foram submetidas à Avaliação Diagnóstica da Fala, considerada padrão-ouro. Resultados: A prevalência de transtornos fonológicos foi de 36,2\%. O teste apresentou sensibilidade de 94,0\%, porém baixa especificidade $(41,1 \%)$. Ao considerar as variações linguísticas como produção normal, o teste mostrou sensibilidade de $86,7 \%$, especificidade de $75,3 \%$, valor preditivo positivo de $66,7 \%$ e valor preditivo negativo de $90,9 \%$. Conclusão: Encontrou-se alta prevalência de transtornos fonológicos. Dado que maioria dos sujeitos não reconheceu alguma figura e o fato de terem ocorrido produções que refletem condições socioculturais, o teste deverá ser revisado para obter maior acurácia na identificação de transtornos fonológicos.
\end{abstract}

Descritores: Transtornos da articulação/epidemiologia; Sensibilidade e especificidade; Prevalência; Programas de rastreamento; Linguística

\section{INTRODUÇÃO}

Muitas áreas da Fonoaudiologia carecem de estudos epidemiológicos de alterações para que se possa conhecer não só a incidência e prevalência, mas também o modo de aparecimento dessas alterações nos indivíduos e avançar no entendimento da interferência dessas alterações na qualidade de $\operatorname{vida}^{(1)}$. Em países com maior tradição em estudos epidemiológicos, como os Estados Unidos, a partir do momento em que se identificou a relevância da taxa de prevalência de distúrbios da comunicação, estruturou-se programas preventivos abrangentes de sensibilização e instrumentação das famílias, equipes de saúde e educação, bem como intervenções específicas no próprio am-

Trabalho realizado no Curso de Mestrado em Ciências da Saúde, Universidade Estadual de Montes Claros - Unimontes - Montes Claros (MG), Brasil. (1) Curso de Odontologia, Universidade Estadual de Montes Claros - Unimontes - Montes Claros (MG), Brasil.

(2) Curso de Medicina e Mestrado em Ciências da Saúde, Universidade Estadual de Montes Claros - Unimontes - Montes Claros (MG), Brasil.

(3) Curso Graduação em Medicina, Universidade Estadual de Montes Claros - Unimontes - Montes Claros (MG), Brasil.

Endereço para correspondência: Luiza Augusta Rosa Rossi-Barbosa. R. São

Marcos, 115, Todos os Santos, Montes Claros (MG), Brasil, CEP: 39400-128.

E-mail: luiza.rossi@unimontes.br

Recebido em: 28/6/2010; Aceito em: 28/10/2010 biente escolar. Porém, apesar dessa relevância epidemiológica, as desordens de fala ainda são pouco exploradas ${ }^{(2)}$.

Dentre esses distúrbios da comunicação está o distúrbio fonológico, que até 1970 recebeu várias denominações: dislalia, atraso de fala, atraso no desenvolvimento da fala, distúrbio articulatório, distúrbio articulatório funcional, desvio de articulação e distúrbio fonológico do desenvolvimento ${ }^{(3,4)}$. Alguns autores utilizam o termo transtorno fonológico ${ }^{(5-7)}$, que consta no Diagnostic and Statistical Manual of Mental Disorders - Fourth Edition (Manual Diagnóstico e Estatístico de Doenças Mentais - Quarta Edição) - DSM IV 315.39 em substituição ao denominado transtorno do desenvolvimento da $\operatorname{articulação} 0^{(8)}$.

O transtorno fonológico é uma alteração na produção da fala e/ou representação mental dos sons de uma língua ${ }^{(9)}$. Pode, então, ser de natureza estritamente fonética (dificuldade na articulação dos sons da fala de ordem motora) ou fonêmica (a forma como a informação dos sons da fala é armazenada e representada no léxico mental, podendo ter base linguística ou cognitiva $)^{(4,6,9,10)}$. É caracterizado pelo uso inadequado de sons, de acordo com a idade e com variações regionais, podendo incluir erros na produção, percepção ou organização dos sons ${ }^{(4)}$.

Um modelo que tem sido muito utilizado na literatura brasileira para a descrição do sistema fonológico da criança é 
o dos processos fonológicos, que diz respeito à simplificação das regras fonológicas que envolvem sequências de sons na pronúncia das palavras ${ }^{(11,12)}$. Na literatura, há três tipos mais citados de processos fonológicos: os de estrutura silábica, os de substituição, e os de assimilação ${ }^{(5)}$. Para o diagnóstico de transtorno fonológico é necessário aplicar testes específicos, nos quais é frequente a utilização de três tipos de provas: imitação, nomeação e fala espontânea ${ }^{(5)}$.

$\mathrm{Na}$ literatura nacional a prevalência de transtorno fonológico varia de $4,2 \%$ a $63,2 \%{ }^{(1-3,12-14)}$, a magnitude da variação pode ser explicada por divergências metodológicas na aferição e na nomenclatura dos distúrbios de fala.

O primeiro teste validado no Brasil para triagem de problemas de fala foi desenvolvido por Goulart ${ }^{(13)}$ e denominado Teste de Rastreamento de Distúrbios Articulatórios de Fala TERDAF. A autora optou por incluir no teste palavras contidas no vocabulário de crianças acima dos 6 anos de idade, de fácil imageabilidade e que representassem de forma equilibrada os fonemas do Português Brasileiro. O teste, realizado com escolares do primeiro ano do ensino fundamental, apresentou boa sensibilidade, porém baixa especificidade, pelo fato de muitas crianças emitirem palavras como fósforo e/ou placa inadequadamente. Ao se retirar esses casos do grupo de crianças com transtornos, a especificidade aumentou. A pesquisadora sugeriu adaptações ao instrumento e aplicações em diferentes grupos populacionais infantis como forma de aprimoramento do teste. As adaptações sugeridas foram: substituição das figuras fósforo e placa ou inclusão de instruções adicionais; na figura do nenê, adotar facilitadores em forma de frases para evocar o vocábulo esperado; incluir uma figura que possibilite a avaliação do fonema /z/.

O objetivo deste trabalho foi conhecer a prevalência dos transtornos fonológicos, por meio dos processos fonológicos, em escolares do primeiro ano do ensino fundamental em uma cidade da região norte de Minas Gerais. Concomitantemente avaliou-se a sensibilidade e especificidade do TERDAF adaptado.

\section{MÉTODOS}

Trata-se de estudo transversal, analítico realizado em Montes Claros - MG, cidade com estimativa de 363.227 habitantes $^{(15)}$, que representa o principal polo urbano da região norte mineira.

Participaram desta pesquisa crianças matriculadas no $1^{\circ}$ ano do ensino fundamental, das 56 escolas municipais e estaduais urbanas de Montes Claros, avaliadas nos meses de maio a agosto de 2009. Para a alocação da amostra, no teste de triagem, empregou-se a técnica da amostragem por conglomerados. As escolas estaduais contabilizaram 96 turmas e as municipais 54, totalizando uma população de aproximadamente 3790 crianças. O cálculo amostral foi definido a partir do nível confiança de $95 \%$, com precisão de $5 \%$ e prevalência estimada de distúrbios de fala de $25 \%$, o valor encontrado foi multiplicado por dois, como fator de correção na amostragem por conglomerado, indicando um número mínimo de 536 crianças. O cálculo para a Avaliação Diagnóstica da Fala (padrão-ouro) considerou a sensibilidade e especificidade es- timadas em pesquisa anterior ${ }^{(13)}$, sendo necessário um mínimo de 227 indivíduos.

Antes da realização do trabalho, foi conduzido um teste piloto com 60 crianças do primeiro ano do ensino fundamental de uma escola estadual do centro da cidade e outra municipal localizada na periferia, com a inserção das seguintes modificações no TERDAF ${ }^{(13)}$ : a figura de um fósforo, para pesquisar o fonema /s/ foi substituída pela escova, a figura de uma placa em um poste foi adaptada por uma placa de trânsito, e para o fonema /3/ foi incluída a gravura de um relógio que também possui o fonema $/ \mathrm{h} /$, o que possibilitou a retirada da figura do rádio.

Os resultados no teste piloto subsidiaram novas adaptações ao instrumento. Verificou-se variação lexical na palavra representada pela figura nenê: 68,3\% disseram bebê e 11,6\% substituíram pela palavra menino. A palavra representada pela figura da placa foi dita por 16,6\% $(\mathrm{n}=10)$ como trânsito, esportes, símbolos. Grande parte do grupo avaliado disse mão ao invés de dedo, havendo necessidade de facilitador para a evocação esperada. Necessitou, também, de facilitador para a emissão da palavra representada pela chave. Seis alunos (10\%) não reconheceram a figura da zebra. As alterações, a partir do teste piloto foram: substituição da figura da placa de trânsito por uma placa de carro, da figura do nenê pela gravura de banana para representar o fonema $/ \mathrm{n} /$, além de figuras melhor representativas para dedo, zebra e chave. Foi incluída, também, a contagem automática de 1 a 10 para facilitar a avaliação do desvio fonético (Anexo 1).

Foram avaliadas 587 crianças do primeiro ano do ensino fundamental de escolas públicas de Montes Claros (MG). Da amostra estudada 61,5\% ( $\mathrm{n}=361)$ estavam em escolas estaduais e 38,5\% ( $n=226)$ municipais, 50,9\% $(n=299)$ eram do sexo masculino. A faixa etária variou de 5 anos e 7 meses a 8 anos e 7 meses; a média de idade foi de 6 anos e 6 meses (DP $\pm 5,06$ ); $91,3 \%$ com idade inferior a 7 anos no momento da triagem; destes 5,1\% tinham menos de 6 anos.

O resultado final da triagem foi classificado como "normal" quando todas as respostas foram corretas, "alterado" quando alguma resposta foi inadequada, e "inconclusivo" quando a criança deixou de falar o nome de uma figura por não tê-la reconhecido ou substituído por outro nome apesar de ter emitido corretamente os fonemas. A aplicação da triagem foi realizada na própria escola por acadêmicos da área da saúde, especialmente treinados.

Uma sub-amostra foi alocada através de seleção aleatória simples, contendo 369 crianças, e estas foram reavaliadas por meio da Avaliação Diagnóstica da Fala ${ }^{(13)}$ para avaliar a sensibilidade e especificidade do teste.

Embora a literatura aborde outros testes diagnósticos mais utilizados, optou-se como padrão-ouro a Avaliação Diagnóstica da Fala para que pudesse ter o mesmo parâmetro da pesquisa de Goulart ${ }^{(13)}$ ao adaptar o teste de rastreamento. Este foi aplicado por fonoaudióloga com experiência clínica. A avaliação constou de repetição de palavras que correspondem a todos os sons do Português e foi solicitado, também, que a criança contasse uma história, a fim de coletar uma amostra de fala espontânea. A profissional considerou o resultado como normal quando todas as produções dos fones do Português Brasileiro 
foram empregadas adequadamente; foi considerado como alterado caso a criança produzisse processos fonológicos tais como apagamento, substituição, adição.

Para verificar a sensibilidade e especificidade utilizou-se dois tipos de análises: o primeiro com o corpus total e o segundo, o corpus sem a variação sociocultural.

Os dados coletados foram codificados e digitados por meio do programa Statistical Package for the Social Sciences - SPSS, versão 15.0, que forneceu os valores de prevalência, sensibilidade, especificidade, valores preditivos positivo e negativo, com os respectivos intervalos de confiança de $95 \%$.

O presente estudo foi aprovado pelo Comitê de Ética em Pesquisa da Universidade Estadual de Montes Claros - Unimontes, sob o número 1236/08. A aplicação dos testes foi realizada mediante assinatura do Termo de Consentimento Livre e Esclarecido (TCLE) pelos pais ou responsáveis. As crianças que apresentaram problemas de fala foram encaminhadas para tratamento especializado. Também foram feitas orientações aos professores das escolas públicas e aos pais dos alunos quanto à prevenção de tais distúrbios.

\section{RESULTADOS}

Entre as crianças testadas, $16,7 \%(\mathrm{n}=98)$ apresentaram fala normal na triagem, 34,8\% $(\mathrm{n}=204)$ apresentaram emissões com alguma alteração e 48,6\% (n=285) não reconheceram alguma figura. Entre as 204 crianças que apresentaram emissões com alguma alteração, as mais prevalentes foram para as figuras da borboleta, folha, coelho e placa, sendo emitida como

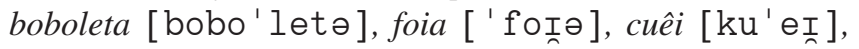

сие̂iи [ku'eIv], сое̂iи [ko'eIv] e praca ['prakə], respectivamente.

A Tabela 1 apresenta, em ordem decrescente, a frequência e exemplos de emissões das 285 crianças que não reconheceram alguma figura.

Entre as 369 crianças da sub-amostra, 140 (37,9\%) foram excluídas por não terem reconhecido alguma figura. Do restante, $146(39,6 \%)$ apresentaram fala normal e $83(22,5 \%)$ fala alterada. A prevalência estimada de transtorno fonológico foi de $36,2 \%$.

A Tabela 2 traz os resultados da triagem comparados com a Avaliação Diagnóstica de Fala (padrão-ouro): a triagem apresentou sensibilidade de 94,0\% (IC 95\%: 88,9\% - 99,1\%) e especificidade de $41,1 \%$ (IC $95 \%: 33,1 \%-49,1 \%$ ); o valor preditivo positivo foi $47,6 \%$ (IC $95 \%$ : $40,0 \%-55,2 \%$ ) e o valor preditivo negativo foi 92,3\% (IC 95\%: 85,9\% - 98,9\%).

Das 229 crianças, 88 falaram boboleta [bobo ' letə] e destas, $76(86,4 \%)$ pronunciaram o fonema medial $/ \mathrm{h} /$ corretamente na palavra carne no teste padrão-ouro. As outras 12 $(13,6 \%)$ crianças o omitiram tanto na palavra carne quanto na fala espontânea, como por exemplo: [ 'poku] para porco, [ ' kodə] para corda, [i' mã ] para irmã. Quanto ao $/ K /$ observou-se que $26(11,4 \%)$ alunos substituíram pelo fonema /y/ ou /i/ em ambas as figuras (do coelho e da folha) e no padrão-ouro algumas pronunciaram corretamente em galho. Em relação à figura da placa, $25(10,9 \%)$ dos alunos substituíram o fonema /r/por/l/, ou seja falaram [ ' prakə], mas no teste diagnóstico algumas emitiram a palavra planta corretamente.

Ao retirar as emissões consideradas como variações socioculturais: [bobo' letə], [ for $ə$ ], [ $k$ ' ' e I ],

Tabela 1. Frequência e exemplos de emissões, sem reconhecimento de figura, realizadas pelas crianças do $1^{\circ}$ ano de escolas públicas do ensino fundamental

\begin{tabular}{|c|c|c|c|}
\hline Palavra & $\mathrm{n}$ & $\%$ & Emissão \\
\hline Zebra & 140 & 23,9 & girafa, "não sei", cavalo, burro, bode, cabra, bezerro, lobo, urso, vaca... \\
\hline Placa & 140 & 23,9 & "não sei", números, letras, quadro, marca, garage \\
\hline Caminhão & 130 & 22,1 & carro, carrinho, carreta, carro de boi \\
\hline Folha & 96 & 16,4 & flor, planta, árvore, mato, pena, tuirô \\
\hline Passarinho & 95 & 16,2 & papagaio, pintinho, pássaro, pato, periquito, pombo, louro, pardal... \\
\hline Violão & 63 & 10,7 & viola, violino, piano, "ioion" \\
\hline Presente & 39 & 6,6 & caixa, caixinha, surpresa, "não sei”, "oidipaca" \\
\hline Dedo & 31 & 5,3 & mão, "soi noni", espinho \\
\hline Sapato & 22 & 3,7 & bota, tênis, botina \\
\hline Coelho & 19 & 3,2 & rato, galo, Páscoa \\
\hline Escova & 15 & 2,6 & pasta \\
\hline Gato & 7 & 1,2 & miau, rato, macaco, ratinho \\
\hline Relógio & 7 & 1,2 & horas \\
\hline Borboleta & 6 & 1,0 & "não sei”, abelha \\
\hline Chave & 6 & 1,0 & ferramenta, revólver, "sai" \\
\hline Maçã & 6 & 1,0 & laranja, abóbora \\
\hline Tesoura & 3 & 0,5 & faca, lápis, \\
\hline Lápis & 1 & 0,2 & lapiseira \\
\hline Cachorro & 1 & 0,2 & cão \\
\hline Banana & 0 & - & \\
\hline
\end{tabular}


Tabela 2. Análise comparativa entre o Teste de Triagem de Fala (TERDAF adaptado) e a Avaliação Diagnóstica da Fala (padrão-ouro) em crianças do $1^{\circ}$ ano de escolas públicas do ensino fundamental

\begin{tabular}{lccc}
\hline \multirow{2}{*}{ TERDAF adaptado } & \multicolumn{3}{c}{ Avaliação Diagnóstica da Fala (padrão-ouro) } \\
\cline { 2 - 4 } & Alterado & Normal & Total $(\mathrm{n})$ \\
\hline Alterado & 78 & 86 & 164 \\
Normal & 5 & 60 & 65 \\
Total $(\mathrm{n})$ & 83 & 146 & 229 \\
\hline
\end{tabular}

[ku'eI U], [ko'eI U ] e [ 'prakə] (Tabela 3), 108 apresentaram triagem alterada e 121 apresentaram triagem normal. A prevalência de transtorno fonológico manteve-se em $36,2 \%$. A Tabela 4 indica a sensibilidade de $86,7 \%$ (IC 95\%: 79,4\% - 94,0\%) e especificidade de 75,3\% (IC 95\%: $68,3 \%-82,3 \%$ ); o valor preditivo positivo de $66,7 \%$ (IC $95 \%$ : $57,8 \%-75,6 \%$ ) e valor preditivo negativo de $90,9 \%$ (IC 95\%: $84,7 \%-95,3 \%)$.

Tabela 3. Frequência e exemplos de emissões realizadas pelas crianças do $1^{\circ}$ ano de escolas públicas do ensino fundamental, consideradas como erros pelos aplicadores do teste de triagem de fala

\begin{tabular}{|c|c|c|c|}
\hline Palavra & $\mathrm{n}$ & $\%$ & Emissão \\
\hline Borboleta & 194 & 33,0 & $\begin{array}{l}\text { [bobo'letə]* [bobou'etə] } \\
{[\text { bole'letə] [bohbo' Ietə] }} \\
{[\text { babu'letə] [popo'letə] }} \\
\text { [pohpo'retə] }\end{array}$ \\
\hline Folha & 116 & 19,8 & 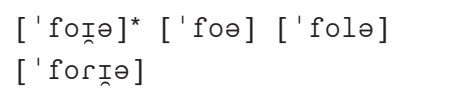 \\
\hline Coelho & 106 & 18,1 & 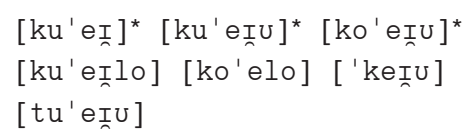 \\
\hline Placa & 79 & 13,5 & [ 'prakə]* ['pakə] ['paklə] \\
\hline
\end{tabular}

* Produções consideradas variações socioculturais

Tabela 4. Análise comparativa entre o Teste de Triagem de Fala (TERDAF adaptado) e a Avaliação Diagnóstica da Fala (padrão-ouro) em crianças do $1^{\circ}$ ano de escolas públicas do ensino fundamental, excluindo as emissões consideradas variações socioculturais

\begin{tabular}{lccc}
\hline \multirow{2}{*}{ Triagem } & \multicolumn{3}{c}{ Avaliação Diagnóstica da Fala (padrão-ouro) } \\
\cline { 2 - 4 } & Alterado & Normal & Total $(\mathrm{n})$ \\
\hline Alterado & 72 & 36 & 108 \\
Normal & 11 & 110 & 121 \\
Total $(\mathrm{n})$ & 83 & 146 & 229 \\
\hline
\end{tabular}

Dentre as 83 crianças que apresentaram alteração de fala no padrão-ouro, $71,1 \%$ apresentaram desvio fonológico e fonético/fonológico e $28,9 \%$ tinham desvio exclusivamente fonético, devido a ceceio anterior e fissura palatina operada.

\section{DISCUSSÃO}

A prevalência do transtorno fonológico de $36,2 \%$ está entre os valores estimados pelos estudos da literatura brasileira, que apontam de $4,2 \%$ a $63,2 \%\left(^{(1-3,12-14)}\right.$. Tal variabilidade talvez possa ser explicada pelos diferentes critérios diagnósticos emprega- dos, pelo número de sujeitos nos estudos, pela utilização de diversas definições desse distúrbio e também pela idade dos indivíduos nas amostras.

Estudo realizado em São Paulo, SP, avaliou o desenvolvimento da fala e linguagem de 2980 crianças de 1 a 11 anos, excluindo aquelas que não ocorrem em conjunção com outras anormalidades, tais como: deficiência mental, paralisia cerebral, deficiências auditivas e outras ${ }^{(2)}$. Nele constatou-se prevalência de 4,2\%. Pesquisa com 2880 crianças em Salvador, BA, em idade entre 6 anos e 6 anos e 11 meses, teve como critérios de exclusão, além de outras, indivíduos que apresentaram alterações na triagem auditiva e as alterações miofuncionais. O valor da prevalência foi de $9,1 \%$, Trabalho realizado em Belo Horizonte, MG, com 95 crianças de escolas infantis da rede particular, mostrou prevalência de $63,2 \%$ mas a média de idade foi de 5 anos e 4 meses $^{(12)}$.

Esta pesquisa tem metodologia semelhante ao estudo com amostra de 184 crianças da primeira série do ensino fundamental cuja prevalência de transtorno fonológico foi de $25,0 \%{ }^{(13)}$. Porém, as idades variaram entre 6 e 12 anos, sendo apenas $27,6 \%$ com idade inferior a 7 anos.

A idade está relacionada a este transtorno. Há diminuição no número de processos fonológicos ao longo dos anos ${ }^{(5,12)}$. $\mathrm{Na}$ faixa dos 6 anos já não se espera encontrar trocas, omissões ou distorções dos sons da fala ${ }^{(16)}$. E há estudos referindo que o desenvolvimento fonológico deve estar praticamente completo aos $5 \operatorname{anos}^{(17,18)}$.

Quanto às porcentagens de desvio fonológico, fonético e fonético/fonológico, resultado semelhante foi encontrado na literatura brasileira: $72,5 \%$ apresentaram diagnóstico de desvio fonológico e $27,5 \%$, desvio fonético ${ }^{(19)}$.

No que se refere à especificidade do teste de triagem, em um primeiro momento mostrou-se baixa, $41,1 \%$, o que pode ser explicado principalmente pela emissão inadequada da palavra borboleta, além de outras como coelho, folha e placa. Ao considerar tais emissões como corretas, devido à variação sociocultural, a especificidade aumentou para 75,3\%. Estes resultados são semelhantes ao estudo anterior, com a mesma metodologia. Nesse estudo, a especificidade inicial do teste foi $22,4 \%$ e ao considerar determinadas palavras como variantes socioculturais e não erros, a especificidade aumentou para $74,1 \%{ }^{(20)}$. Para validação de instrumentos é importante considerar a faixa etária e os regionalismos em relação à avaliação de fala e linguagem ${ }^{(21)}$.

Quanto ao apagamento do fonema medial /h/ na palavra borboleta, 86,4\% repetiram corretamente este fonema no teste diagnóstico. Tal fato mostra que a figura da borboleta no teste de triagem deverá ser substituída.

A troca do fonema $/ K /$ por $/ y /$ em coelho e folha também foi observada na repetição da palavra galho durante a avaliação diagnóstica e na fala espontânea fizeram substituições, como por exemplo: [ 'mi ] ou [ 'mi İU] para milho, [pa' I IasU ] para palhaço, [ku' IE ] para colher, [ 'OIU ] para óleo, [mu ' ${ }_{N} \varepsilon$ ] para mulher. Esta líquida surge tardiamente no desenvolvimento das crianças e são confundidas com a semi$\operatorname{vogal} / \mathrm{w} /$ no Inglês e com o /y/ no Português ${ }^{(22)}$.

Sobre a substituição do fonema $/ \mathrm{r} /$ por $/ \mathrm{l} / \mathrm{em}$ placa durante a triagem e planta na avaliação diagnóstica, cujas emissões 
foram [ 'prakə] e [ 'prãtə], respectivamente, é provável que tal fato esteja relacionado a questões sociais, classificadas como variações socioculturais ${ }^{(13,20)}$. Em estudo com crianças residentes na cidade de São Paulo, observou-se que alguns processos fonológicos foram produtivos até os 7 anos e se restringiram, praticamente, à substituição da líquida /1/ pela líquida /r/ em encontros consonantais, o que é bastante comum em populações de baixa renda ${ }^{(23)}$.

Mas, curiosamente, na fala espontânea algumas dessas crianças emitiram palavras como atlético e bicicleta corretamente. Porém, nem todas produziram palavras com a líquida /1/ em grupos consonantais durante a fala espontânea. Essa é uma limitação do método de coleta de dados, que dificulta o controle dos estímulos a serem emitidos, de tal modo que nem todos os sons da língua aparecem na amostra ${ }^{(24)}$. O inverso também pode acontecer, crianças com distúrbio de fala produzirem corretamente o som numa palavra e apresentarem substituições e omissões no discurso espontâneo ${ }^{(25)}$.

A prova de fala espontânea depende do engajamento da criança na proposta, o que pode interferir na qualidade do discurso $^{(5)}$. Talvez fique mais fácil solicitar à criança que conte uma história a partir da observação de desenhos temá$\operatorname{ticos}^{(26,27)}$. Sugere-se que contenham as líquidas /1/ e /r/ em grupos consonantais e $/ K /$ : bruxa, ladrão, planta, bicicleta, flor, flauta, palhaço.

A readaptação do teste foi proposta a fim de obter protocolo que considere as variações socioculturais relacionadas ao meio no qual a criança está inserida, tanto em relação às figuras quanto aos vocábulos-alvo ${ }^{(20)}$. Portanto, a figura da borboleta poderá ser substituída pela de uma porta, porco ou barco. Quanto à figura da placa, pelo fato de algumas crianças terem emitido correta e espontaneamente o /1/ em encontro consonantal na palavra bicicleta, tal figura poderá substituí-la.

Um aspecto que merece ser comentado refere-se ao fato de $48,6 \%$ não terem reconhecido alguma figura na triagem. Este índice foi maior do que o encontrado em estudo no qual 10,7\% não souberam reconhecer alguma das ilustrações apresentadas no teste ${ }^{(20)}$. Nesse aspecto, por acreditar que a palavra zebra não faz parte do léxico das crianças pesquisadas, sugere-se substituir pela figura de uma cobra. Quanto ao fonema /z/ o teste consta de figuras tais como presente e tesoura, mas talvez pudesse inserir a representação do número zero. Para a gravura do caminhão, dentre as palavras consideradas inconclusivas, a maioria emitiu carro, o que indica a necessidade de se inserir uma gravura que represente de forma prototípica um caminhão.

\section{CONCLUSÃO}

A taxa de prevalência de transtorno fonológico de 36,2\% está estimada na literatura brasileira.

$\mathrm{O}$ teste de triagem analisado mostrou, após adequação da variação sociocultural, valores satisfatórios de sensibilidade, especificidade, valor preditivo positivo e valor preditivo negativo, o que o torna recomendado para utilização clínica e epidemiológica nas áreas da fala e linguagem e ser aplicado por profissionais da educação e da saúde.

Sabe-se que uma triagem deve levar apenas alguns minutos para ser realizado e ter baixo custo para ser aplicada num grande número de pessoas. O teste estudado é simples e sua aplicação tem duração média de um minuto e meio.

Estudos posteriores propiciarão verificar a acurácia do teste após sofrer as modificações propostas. Existe também a possibilidade de utilização de outra avaliação diagnóstica (padrão-ouro) para comparação, por exemplo, o Teste de Linguagem Infantil na Área de Fonologia (ABFW), bem como a utilização de figuras temáticas para a fala espontânea.

É necessário aprimorar a metodologia de avaliação para diagnósticos dos distúrbios da comunicação humana; realizar estudos epidemiológicos nas escolas e ambientes mais próximos das pessoas; aferir condições e qualidade de vida para promover a saúde da população de referência.

\begin{abstract}
Purpose: To identify the prevalence of phonological disorders and to verify the sensitivity and specificity of the adapted Speech Disorder Screening Test (TERDAF) in first grade students of elementary school in Montes Claros, MG (Brazil). Methods: Children were randomly assigned into cluster sampling in 56 public schools in the city. We assessed 587 children with a mean age of 6 years and 6 months using the adapted TERDAF; to verify the test's sensitivity and specificity, 229 of them were also assessed using the Speech Diagnosis Assessment, considered golden standard method. Results: The prevalence of phonological disorders was $36.2 \%$. The test presented sensitivity of $94.0 \%$, but low specificity (41.1\%). Considering the linguistic variations as normal production, the test had sensitivity of $86.7 \%$, specificity of $75.3 \%$, positive predictive value of $66.7 \%$ and negative predictive value of $90.9 \%$. Conclusion: A high prevalence of phonological disorders was found. Due to the fact that most subjects did not recognize some picture and also that productions reflecting socio-cultural conditions occurred, the test must be further reviewed in order to be more accurate in the identification of phonological disorders.
\end{abstract}

Keywords: Articulation disorders/epidemiology; Sensitivity and specificity; Prevalence; Mass screening; Linguistics 


\section{REFERÊNCIAS}

1. Campos FR, Rabelo AT, Friche AA. Prevalência de alterações fonoaudiológicas em crianças de 5 a 9 de idade de escolas particulares [Internet]. In: $16^{\circ}$ Congresso de Fonoaudiologia; 2008 Set 24-27; Campos do Jordão. Anais eletrônicos. São Paulo: SBFa; 2008 [citado 2009 Jul 22]. Disponível em: http://www.sbfa.org.br/portal/anais2008/ resumos/R1330-2.pdf

2. Andrade CR. Prevalência das desordens idiopáticas da fala e da linguagem em crianças de um a onze anos de idade. Rev Saude Publica. 1997;31(5):495-501.

3. Cavalheiro LG, Keske-Soares M. Prevalência do desvio fonológico em crianças de 4 a 6 anos de idade. Pró-Fono Revista de Atualização Científica [Internet] 2008;20(Sup1):11-3 [Apresentado no II Congresso Brasileiro de Fonoaudiologia e Genética dos Distúrbios da Comunicação; 2008; Fortaleza, Ceará]. [citado 2009 Jul 30]. Disponível em: www.revistaprofono.com.br

4. Wertzner HF. Fonologia: desenvolvimento e alterações. In: Fernandes FD, Mendes BC, Navas AL, organizadores. Tratado de fonoaudiologia. 2a ed. São Paulo: Roca; 2009, p. 281-290.

5. Wertzner HF, Papp AC, Galea DE. Provas de nomeação e imitação como instrumentos de diagnóstico do transtorno fonológico. Pró-Fono. 2006;18(3):303-12.

6. Castro MM, Wertzner HF. Estimulabilidade e tipos de erro de fala. Rev Soc Bras Fonoaudiol. 2006;11(1):1-9.

7. Wertzner HF, Pagan LO, Galea DE, Papp AC. Características fonológicas de crianças com transtorno fonológico com e sem histórico de otite média. Rev Soc Bras Fonoaudiol. 2007;12(1):41-7.

8. American Psychiatric Association. Diagnostic and statistical manual of mental disorders - 4th ed. (DSM-IV) [Internet] [cited 2010 Mar 2] Available from: http://www.psych.org/mainmenu/research/dsmiv.aspx.

9. Gierut JA. Treatment efficacy: functional phonological disorders in children. J Speech Lang Hear Res. 1998;41(1):S85-100.

10. Lima R. Alterações nos sons da fala: o domínio dos modelos fonéticos. Rev. Saber (e) Educar. 2008;13:149-57.

11. Wertzner HF, Rosal CA, Pagan LO. Ocorrência de otite média e infecções de vias aéreas superiores em crianças com distúrbio fonológico. Rev Soc Bras Fonoaudiol. 2002;7(1):31-7.

12. Vitor RM, Cardoso-Martins C. Desenvolvimento fonológico de crianças pré-escolares da Região Noroeste de Belo Horizonte. Psicol Rev (Belo Horizonte). 2007;13(2)383-98.

13. Goulart BN. Validação de teste de rastreamento de distúrbios articulatórios de fala em crianças de $1^{\text {a }}$ série do ensino fundamental público. [dissertação] Porto Alegre: Universidade Federal do Rio Grande do Sul; 2002.
14. Patah LK, Takiuchi N. Prevalência das alterações fonológicas e uso dos processos fonológicos em escolares aos 7 anos. Rev CEFAC. 2008;10(2):158-67.

15. Instituto Brasileiro de Geografia e Estatística - IBGE. Estimativas de população [Internet]. 2009 [citado 2010 Mar 2]. Disponível em: http://www.ibge.gov.br/home/estatistica/populacao/estimativa2009/ POP2009_DOU.pdf

16. Pagan LO, Wertzner HF. Intervenção no distúrbio fonológico através dos pares mínimos com oposição máxima. Pro Fono. 2002;14(3:) 313-24.

17. Ribas LP. Sobre a aquisição do onset complexo. In: Lamprecht RR, Bonilha GF, Freitas GC, Matzenauer CL, Mezzomo CL, Oliveira CC et al. Aquisição fonológica do português: perfil de desenvolvimento e subsídios para terapia. Porto Alegre: Artmed; 2004. p.151-64.

18. Mezzomo CL, Ribas LP. Sobre a aquisição das líquidas. In: Lamprecht RR, Bonilha GF, Freitas GC, Matzenauer CL, Mezzomo CL, Oliveira $\mathrm{CC}$ et al. Aquisição fonológica do português: perfil de desenvolvimento e subsídios para terapia. Porto Alegre: Artmed, 2004. p.95-112.

19. Santos GG, Melo PD, Diniz JM, Teixeira GP. A importância do diagnóstico diferencial das alterações de fala: enfoque fonológico. J Bras Fonoaudiol. 2003;4(16):186-92.

20. Goulart BN, Ferreira J. Teste de rastreamento de alterações de fala para crianças. Pró-Fono. 2009;21(3):231-6.

21. Goulart BNG, Chiari BM. Testes de rastreamento x testes de diagnóstico: atualidades no contexto da atuação fonoaudiológica. PróFono. 2007;19(2):223-32.

22. Pagan LO, Wertzner HF Análise acústica das consoantes líquidas do Português Brasileiro em crianças com e sem transtorno fonológico. Rev Soc Bras Fonoaudiol. 2007;12(2):106-13.

23. Wertzner H. Estudo da aquisição do sistema fonológico: o uso de processos fonológicos em crianças de três a sete anos. Pró-Fono. 1995;7(1):21-6.

24. Mota HB. Terapia fonoaudiológica para os desvios fonológicos. Rio de Janeiro: Revinter; 2001.

25. Klein HB, Liu-Shea M. Between-word simplification patterns in the continuous speech of children with speech sound disorders. Lang Speech Hear Serv Sch. 2009;40(1):17-30.

26. Baldi VG, Homem FC. Caracterização de desvios fonológicos na fala de crianças institucionalizadas de 6 a 7 anos. J Bras Fonoaudiol. 2004;5(18):22-34.

27. Barrera SD, Maluf MR. Variação linguística e alfabetização: um estudo com crianças da primeira série do ensino fundamental. Psicol Esc Educ. 2004;8(1):35-46. 
Anexo 1. TERDAF nodificado

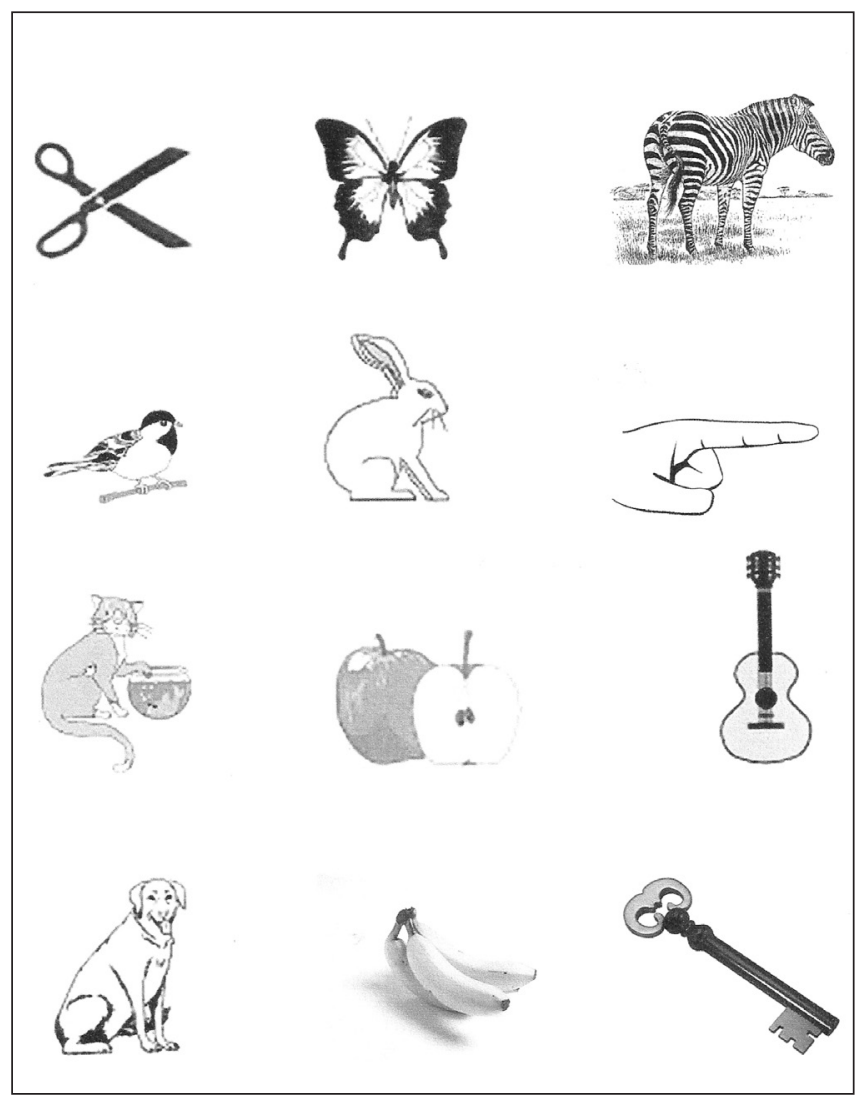

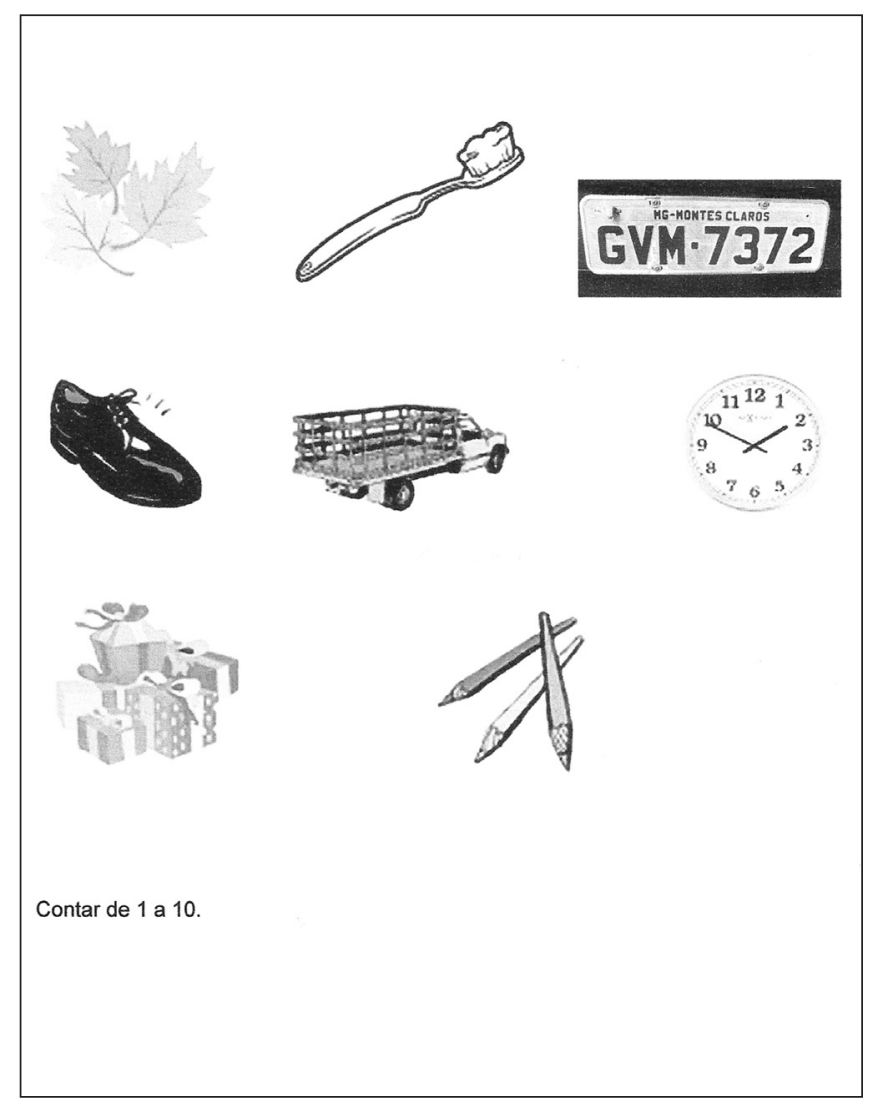

